\title{
Superconducting State Parameters of Bulk Amorphous Alloys
}

\author{
Aditya M. Vora* \\ Humanities and Social Science Department, S.T.B.S. College of Diploma Engineering, \\ Shri Swami Atmanand Sarswati Vidya Sankul, Opp. Kapodra Police Station, \\ Varachha Road, Surat 395 006, Gujarat, India
}

(Received 13 January 2012; published online 29 December 2012)

\begin{abstract}
Well recognized empty core (EMC) pseudopotential of Ashcroft is used to investigate the superconducting state parameters viz; electron-phonon coupling strength $\lambda$, Coulomb pseudopotential $\mu^{*}$, transition temperature $T_{C}$, isotope effect exponent $\alpha$ and effective interaction strength $N_{O} V$ of some $\left(\mathrm{Ni}_{33} \mathrm{Zr}_{67}\right)_{1-x} \mathrm{~V}_{x}$ $(x=0,0.05,0.1,0.15)$ bulk amorphous alloys. We have incorporated five different types of local field correction functions, proposed by Hartree (H), Taylor (T), Ichimaru-Utsumi (IU), Farid et al. (F) and Sarkar et al. (S) to show the effect of exchange and correlation on the aforesaid properties. Very strong influence of the various exchange and correlation functions is concluded from the present study. The $T_{C}$ obtained from Sarkar et al. (S) local field correction function are found an excellent agreement with available theoretical data. Quadratic $T_{C}$ equation has been proposed, which provide successfully the $T_{C}$ values of bulk amorphous alloys under consideration. Also, the present results are found in qualitative agreement with other such earlier reported data, which confirms the superconducting phase in the s bulk amorphous alloys.
\end{abstract}

Keywords: Pseudopotential, Superconducting state parameters (SSP), Bulk amorphous alloys.

PACS numbers: 61.43.Dq; 71.15.Dx; 74.20. - z; 74.70.Ad

\section{INTRODUCTION}

During last several years, the superconductivity remains a dynamic area of research in condensed matter physics with continual discoveries of novel materials and with an increasing demand for novel devices for sophisticated technological applications [1-10]. There are very few scattered attempts to study the superconducting state parameters of bulk amorphous alloys based on model potential [7-10]. Hence, we thought it worthwhile to undertake the investigation of the superconducting state parameters of $\left(\mathrm{Ni}_{33} \mathrm{Zr}_{67}\right)_{1-x} \mathrm{~V}_{x} \quad(\mathrm{x}=0$, $0.05,0.1,0.15$ ) bulk amorphous alloys on the basis of well recognized empty core (EMC) pseudopotential of Ashcroft [11]. We have employed five different types of local field correction functions proposed by Hartree $(\mathrm{H})$ [12], Taylor (T) [13], Ichimaru-Utsumi (IU) [14], Farid et al. (F) [15] and Sarkar et al. [16] to show the effect of the exchange and correlation on the aforesaid properties. For the investigations of electron-phonon coupling strength $\lambda$, Coulomb pseudopotential $\mu^{*}$, transition temperature $T_{C}$, isotope effect exponent $\alpha$ and effective interaction strength $N_{0} V$ for $\left(\mathrm{Ni}_{33} \mathrm{Zr}_{67}\right)_{1-x} \mathrm{~V}_{x}(x=0,0.05$, $0.1,0.15)$ bulk amorphous alloys, we have extended the way followed by McMillan for metals [6-10].

\section{THEORETICAL METHODOLOGY}

The mathematical expressions used for the present investigation of electron-phonon coupling strength $\lambda$, Coulomb pseudopotential $\mu^{*}$, transition temperature $T_{C}$, isotope effect exponent $\alpha$ and effective interaction strength $N_{0} V$ of $\left(\mathrm{Ni}_{33} \mathrm{Zr}_{67}\right)_{1-x} \mathrm{~V}_{x} \quad(x=0,0.05,0.1,0.15)$ bulk amorphous alloys are [6-10].

$$
\lambda=\frac{12 m_{b} Z}{M\left\langle\omega^{2}\right\rangle_{0}^{1}} X^{3}|W(X)|^{2} d X
$$

$$
\begin{gathered}
\mu^{*}=\frac{\frac{m_{b}}{\pi k_{F}} \int_{0}^{1} \frac{d X}{\varepsilon(X)}}{1+\frac{m_{b}}{\pi k_{F}} \ln \left(\frac{E_{F}}{20 \theta_{D}}\right) \int_{0}^{1} \frac{d X}{\varepsilon(X)}} \\
T_{C}=\frac{\theta_{D}}{1.45} \exp \left[\frac{-1,04(1+\lambda)}{\lambda-\mu^{*}(1+0.62 \lambda)}\right], \\
\alpha=\frac{1}{2}\left[1-\left(\mu^{*} \ln \frac{\theta_{D}}{1.45 T_{C}}\right)^{2} \frac{1+0.62 \lambda}{1.04(1+\lambda)}\right] \\
N_{O} V=\frac{\lambda-\mu^{*}}{1+10 \lambda / 11}
\end{gathered}
$$

Where $m_{b}$ is the band mass, $M$ the ionic mass, $\Omega_{0}$ the atomic volume, $k_{F}$ the Fermi wave vector, $W(X)$ the screened pseudopotential, $E_{F}$ the Fermi energy, $\varepsilon(X)$ the modified Hartree dielectric function [4] and $\left\langle\omega^{2}\right\rangle$ the averaged square phonon frequency, respectively. The $\left\langle\omega^{2}\right\rangle$ is calculated using the relation given by Butler [17] i.e. $\left\langle\omega^{2}\right\rangle=0.69 \theta_{D}$, where $\theta_{D}$ is the Debye temperature.

\section{RESULTS AND DISCUSSION}

The input parameters used in the present investigation are given in Table 1. While Fig. 1-5 shows the presently calculated values of the superconducting state parameters along with other such findings [4].

Table 1 - Input parameters and other constants

\begin{tabular}{|c|c|c|c|c|c|}
\hline Superconductors & $Z$ & $r_{\subset}(\mathrm{au})$ & $\begin{array}{c}\Omega o \\
(\mathrm{au})^{3}\end{array}$ & $\begin{array}{c}M \\
(\mathrm{amu})\end{array}$ & $\theta_{D}(\mathrm{~K})$ \\
\hline$\left(\mathrm{Ni}_{33} \mathrm{Zr}_{67}\right)_{1} \mathrm{~V}_{0}$ & 3.34 & 1.4584 & 129.76 & 80.49 & 343.47 \\
\hline$\left(\mathrm{Ni}_{33} \mathrm{Zr}_{67}\right)_{0.95} \mathrm{~V}_{0.05}$ & 3.42 & 1.3988 & 127.95 & 79.01 & 345.30 \\
\hline$\left(\mathrm{Ni}_{33} \mathrm{Zr}_{67}\right)_{0.90} \mathrm{~V}_{0.10}$ & 3.51 & 1.3457 & 126.14 & 77.54 & 347.12 \\
\hline$\left(\mathrm{Ni}_{33} \mathrm{Zr}_{67}\right)_{0.85} \mathrm{~V}_{0.15}$ & 3.59 & 1.2918 & 124.33 & 76.06 & 348.95 \\
\hline
\end{tabular}

\footnotetext{
*voraam@gmail.com
} 


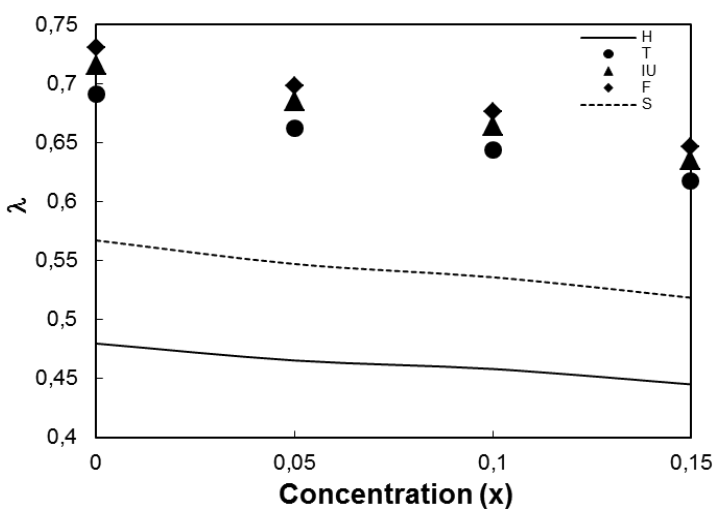

Fig. 1 - Variation of electron-phonon coupling strength $(\lambda)$ with V-concentration $x$ (at. \%)

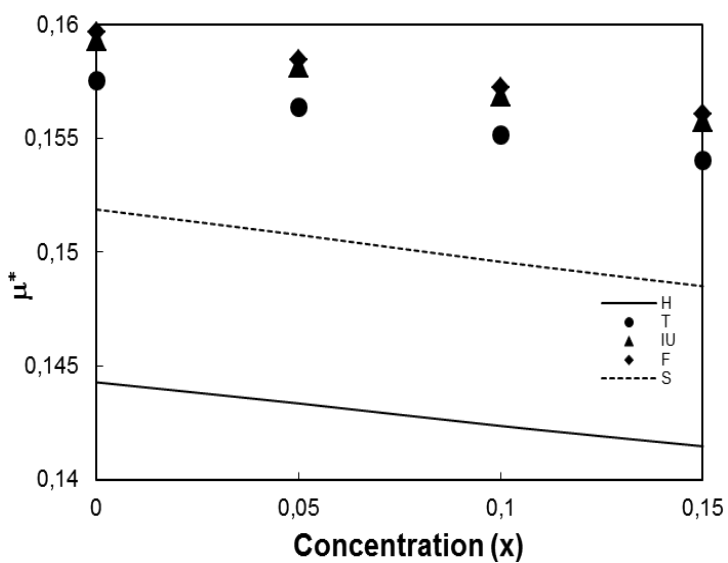

Fig. 2 - Variation of Coulomb pseudopotential $\left(\mu^{*}\right)$ with Vconcentration $x$ (at. \%)

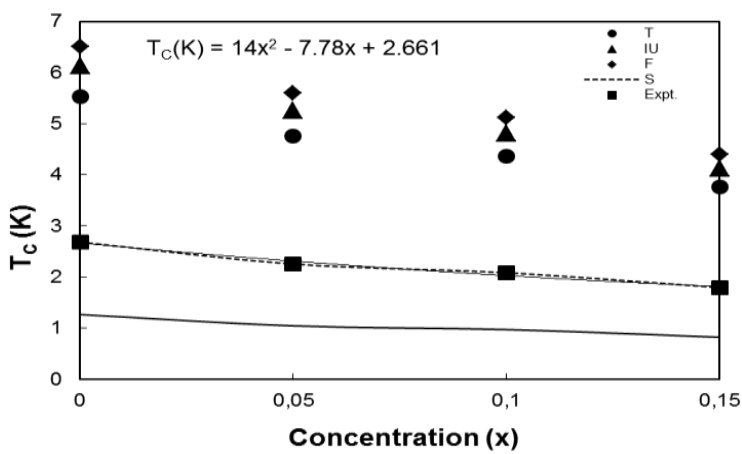

Fig. 3-Variation of transition temperature $\left(T_{C}\right)$ with Vconcentration $x$ (at \%)

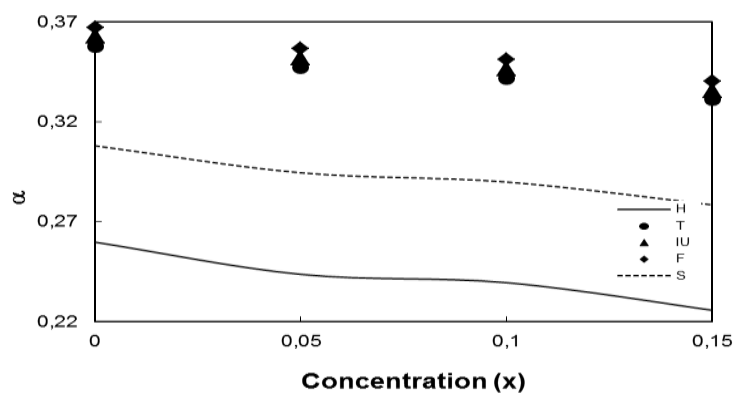

Fig. 4-Variation of isotope effect exponent $(\alpha)$ with Vconcentration $x$ (at. \%)

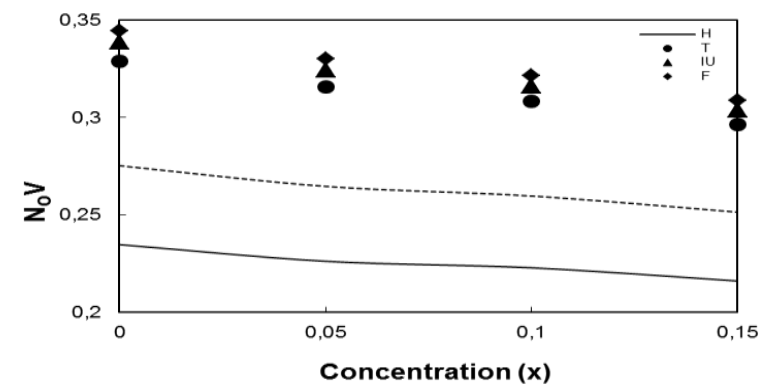

Fig. 5 - Variation of effective interaction strength (NoV) with V-concentration $x$ (at \%)

It is seen that among all the six screening functions, the screening function due to Hartree [12] gives the minimum value of the superconducting state parameters while the screening function due to Farid et al. (F) [15] gives the maximum value. The numerical values of the aforesaid properties are found to be quite sensitive to the selection of the local field correction function and showing a significant variation with the change in the function. With respect to the static $\mathrm{H}$-dielectric function [12] the influence of various local field correction functions on $\lambda$ is $16.53 \%-52.25 \%$. Such influence on $\mu^{*}$ is $4.97 \%-10.66 \%$. These changes in $\lambda$ and $\mu^{*}$ make drastic variation on $T_{C}, \alpha$ and $N_{o} V$. It is also observed from the Fig. 1 that $\lambda$ goes decreasing from the values of $0.7302 \rightarrow 0.4459$ as the concentration ' $x$ ' of ' $\mathrm{V}$ ' is increased from $0.0 \rightarrow 0.15$. The decrease in $\lambda$ with concentration ' $x$ ' of ' $V$ ' shows a gradual transition from weak coupling behaviour to intermediate coupling behaviour of electrons and phonons, which may be attributed to an increase of the hybridization of sp-d electrons of ' $V$ ' with increasing concentration ( $Z$ ). This may also be attributed to the increase role of ionic vibrations in the V-rich region. It is observed from the Fig. 2 that, $\mu^{*}$ lies between 0.14 and 0.16 , which is in accordance with McMillan [6], who suggested $\mu^{*} \approx 0.13$ for transition metals. Present results show the weak dependence of $\mu^{*}$ on the local field correction functions.

Fig. 3 contains calculated values of the transition temperature $T_{C}$ computed from the various forms of the local field correction functions along with experimental [4] findings. The present results obtained from the Slocal field correction functions are found in good agreement with available experimental [4] data. The presently computed $T_{C}$ deviates in the range of $0.02 \%$ $148.80 \%$ from the experimental findings [4]. It is seen that $T_{C}$ is quite sensitive to the local field correction functions, and the results of $T_{C}$ by using S-screening are in best agreement with experimental data [4] for bulk amorphous alloys under investigation, as the relevant curves for S-screening almost overlaps the experimental curves. It is also seen from the graphical nature, $T_{C}$ decreases considerably with increasing Vconcentration $(x)$. The composition dependence can be described by polynomial regression of the data obtained for S-screening for different values of the concentration $x$, which yields

$$
T_{C}(K)=14 x^{2}-7.78 x+2.661
$$

The graph of the fitted $T_{C}$ equation is displayed in Fig. 4, which indicates that $T_{C}$ decreases considerably 
with increasing ' $V$ ' content with a slope $d T_{C} / \mathrm{dx}=7.78$. Wide extrapolation predicts a $T_{C}=2.661 \mathrm{~K}$ for the hypothetical case of 'amorphous pure $\mathrm{Ni}_{33} \mathrm{Zr}_{67}$ alloy. This quadratic relation is found in qualitative agreement with those given by Sharma et al. [7].

The values of the isotope effect exponent $\alpha$ for $\left(\mathrm{Ni}_{33} \mathrm{Zr}_{67}\right)_{1-x} \mathrm{~V}_{x} \quad(x=0,0.05,0.1,0.15)$ bulk amorphous alloys are seen Fig. 4 . The computed values of $\alpha$ show a weak dependence on the dielectric screening function. Since the experimental value of $\alpha$ has not been reported in the literature so far, the present data of $\alpha$ may be used for the study of ionic vibrations in the superconductivity of alloying substances. The values of the effective interaction strength $N o V$ are depicted in Fig. 5 for different local field correction functions. It is observed that the magnitude of $N o V$ shows that the $\left(\mathrm{Ni}_{33} \mathrm{Zr}_{67}\right)_{1-x} \mathrm{~V}_{x}$ $(x=0,0.05,0.1,0.15)$ bulk amorphous alloys under investigation lie in the range of weak to intermediate superconductors.

It is observed that this simple methodology successfully explains superconducting behaviour of bulk amorphous alloys without requiring the solution of the Dirac equation for many body problem or estimation of various interactions as required in ab-initio pseudopotential theory. In the present work superconducting properties of bulk amorphous alloys have been determined in the BCS-Eliashberg-McMillan framework. It is observed that addition of ' $V$ ' as the third element $(M)$ to a binary metallic systems $\left(\mathrm{Ni}_{33} \mathrm{Zr}_{67}\right)$ causes the parameters $\lambda, T_{C}, \alpha$ and $N_{o} V$ to decrease, and the Coulomb pseudopotential $\left(\mu^{*}\right)$ to increase with concentration of the third element $(M)$, showing that the presence of the third element $(M)$ causes suppression of superconducting behaviour of the alloy. The decrease in $T_{C}$ with increasing concentration of the third element $(M)$ may be attributed to the modifications in the density of states at the Fermi level $N\left(E_{F}\right)$, and probable changes in the band structure of the alloy due to addition of the third element $(M)$ [7]. Both specific heat measurements and band structure calculation [1-4] reveal the decrease in density of states at $E_{F}$ with the addition of the third element $(M)$. Since, $T_{C}$ is related to the modifications of density of states (DOS) at $E_{F}, N\left(E_{F}\right)$, decrease in $T_{C}$ can be related to the modifications of DOS at the Fermi level, $N\left(E_{F}\right)$ [7]. It is also observed that superconductivity persists only for small values of $x$ (i.e. $x \leq 0.15$ ) which is because the third element $(M)$ considered here are all $3 d$-transition metals which have smaller band width and stronger localized character than $\mathrm{Zr}$, thus they causes narrowing of bands in bulk system [7]. These narrow bands have magnetic instabilities which prevent superconductivity as suggested by Allen and Dynes [18].

\section{CONCLUSIONS}

Lastly we concluded that, the H-local field corrections when used with EMC model potential provide the best explanation for superconductivity in $\left(\mathrm{Ni}_{33} \mathrm{Zr}_{67}\right)_{1-x} \mathrm{~V}_{x} \quad(x=0,0.05,0.1,0.15)$ bulk amorphous superconductors. The values of $\lambda$ and $T_{C}$ show an appreciable dependence on the local field correction function, whereas for $\mu^{*}, \alpha$ and $N o V$ a weak dependence is observed. The magnitude of $\lambda, \alpha$ and $N o V$ values shows that, the bulk amorphous alloys are weak to intermediate superconductors. Quadratic $T_{C}$ equation has been proposed, which provide successfully the $T_{C}$ values of the bulk amorphous alloys under consideration. In the absence of experimental data for $\alpha$ and $N o V$, the presently computed values may be considered to form reliable data for these bulk systems, as they lie within the theoretical limits of the Eliashberg-McMillan formulation. The comparisons of presently computed results of the superconducting state parameters (SSP) of the $\left(\mathrm{Ni}_{33} \mathrm{Zr}_{67}\right)_{1-x} \mathrm{~V}_{x} \quad(x=0,0.05,0.1,0.15)$ bulk amorphous alloys with available experimental findings are highly encouraging, which confirms the applicability of the EMC model potential and different forms of the local field correction functions. Such study on superconducting state parameters (SSP) of other multi component metallic alloys is in progress.

\section{REFERENCES}

1. U. Mizutani, C. Mishima, T Goto, J. Phys. Cond. Mat. 1, 1831 (1989).

2. R. Zehringer, P. Oelhafen, H.-J. Guntherodt, Y. Yamada, U. Mizutani, Mater. Sci. Eng. 99, 317 (1988).

3. U. Mizutani, U. Mizutani, C. Mishima, Solid State Commun. 62, 641 (1987).

4. Y. Yamada, Y. Itoh, U. Mizutani, Mater. Sci. Eng. 99, 289 (1988).

5. A.V. Narlikar, S.N. Ekbote, Superconductivity and Superconducting Materials, (New Delhi - Madras: South Asian Publishers: 1983)

6. W.L. McMillan, Phys. Rev. 167, 331 (1968).

7. S. Sharma, H. Khan, K.S. Sharma, Czech. J. Phys. 55, 1005 (2005).

8. P. Chatterjee, Can. J. Phys. 58, 1383 (1980).

9. Aditya M. Vora, J. Supercond. Novel Magn. 24, 2065 (2011)

10. Aditya M. Vora, J. Non-Cryst. Sol. 357, 2039 (2011).

11. N.W. Ashcroft, Phys. Lett. 23, 48 (1966).

12. W. Harrison, Elementary Electronic Structure (Singapore: World Scientific: 1999).

13. R. Taylor, J. Phys. F: Met. Phys. 8, 1699 (1978).

14. S. Ichimaru, K. Utsumi, Phys. Rev. B 24, 7385 (1981).

15. B. Farid, V. Heine, G. Engel, I.J. Robertson, Phys. Rev. B 48, 11602 (1993).

16. A. Sarkar, D.S. Sen, S. Haldar, D. Roy, Mod. Phys. Lett. B 12, 639 (1998)

17. W.H. Butler, Phys. Rev. B 15, 5267 (1977).

18. P.B. Allen, M.L. Cohen, Phys. Rev. 187, 525 (1969). 